\title{
CLOSED FORM BOUND-STATE PERTURBATION THEORY
}

\author{
OLLIE J. ROSE \\ SDC Integrated Services, Inc. \\ Hampton \\ Virginia 23666 \\ CARL G. ADLER \\ Physics Department \\ East Carolina University \\ Greenville \\ North Carolina 27834 \\ U. S. A. \\ (Received January 23, 1979) \\ (In Revised Form December 18, 1979)
}

ABSTRACT: The perturbed Schrödinger eigenvalue problem for bound states is cast Into Integral form using Green's Functions. A systematic algorithm is developed and applied to the resulting equation giving rise to approximate solutions expressed as functions of the given perturbation parameter. As a by-product, convergence radil for the traditional Rayleigh-Schrödinger and Brillouin-Wigner perturbation theories emerge in a natural way. KEY WORDS AND PHRASES: Closed Form Perturbation Theory. 1980 MATHEMATICS SUBJECT CLASSIFICATION CODES: $81 A 10$ 


\section{INTRODUCTION}

The method of Green's Functions is a powerful and widely used tool for obtaining analytical solutions to boundary value problems arising in diverse areas of physics; most notably in potential theory. [1, 2] The Green's Function approach is also extensively used to establish approximation methods in quantum mechanical collision theory. [3] However the use of closed form Green's Functions as a basis for a perturbation treatment of bound state quantal problems has not been exploited. [4,5] In this paper, we view the notions of Green's Function and integral representations as unifying concepts from which conventional bound-state perturbation theory can be derived. From this approach, we are able to derive various properties such as convergence radii for the different perturbation approximations. In addition, there are obvious advantages to obtaining approximate results for energy and wavefunctions in a closed form (see the Appendix in Section 5 for an example).

Although the arguments in this paper are restricted to bound-state solutions of the time-independent Schrödinger equation, there is generality in two ways. First, the results apply to any number of dimensions and to essentially arbitrary potentials; secondly, there is an easy generalization to almost any linear differential equation of elliptic type.

The general equation we wish to solve is

$$
\mathrm{H} \psi(r)=\mathrm{E} \psi(r)
$$

where $H$ is defined as usual and $\psi(r)$ is defined on some domain $\Omega$ with boundary $\partial \Omega$. We seek a solution to eq. (1.1) defined over $\Omega$ and subject to boundary condition $\psi(r)=0$, for $r$ belonging to $\partial \Omega$. Further we wish to know the allowed values of the unknown parameter $\mathrm{E}$. We assume that $\Omega$ is an open, simply connected subset of Euclidean space with piece-wise smooth boundary. We further 
suppose that all eigenstates under discussion are non-degenerate. No essential differences are needed to treat the degenerate case.

The essence of perturbation theory is to suppose that $\mathrm{H}$ can be written as $\mathrm{H}_{0}+\varepsilon \mathrm{H}_{1}$, where $\varepsilon$ is a small parameter and further that we know the solution to

$$
\mathrm{H}_{0} \phi=W \phi \text {. }
$$

We wish to exploit this knowledge to approximate the solutions to eq. (1.1)

written in the form

$$
\left(\mathrm{H}_{0}+\varepsilon \mathrm{H}_{1}\right) \psi=\mathrm{E} \psi \text {. }
$$

We adopt the following notational definitions.
a) $\phi_{n}$ - unperturbed wavefunction for the $n \frac{\text { th }}{\text { eigenstate }}$
b) $w_{n}$ - energy eigenvalue corresponding to $\phi_{n}$

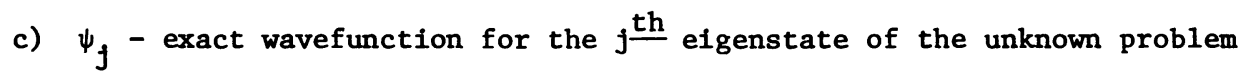
d) $E_{j}$ - exact energy eigenvalue corresponding to $\psi_{j}$

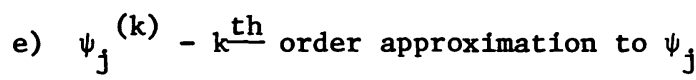

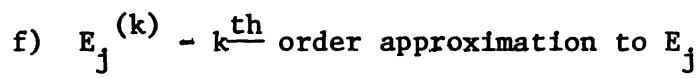

\section{STATEMENT OF RELEVANT RESULTS FROM ANALYSIS}

2.1 GREEN'S IDENTITIES: Let $\Omega$ be an open simply connected region of $R^{\nu}$ with sectionally smooth boundary $\partial \Omega$. Let $L(\bar{x})$ be a differential operator of the form

$$
L(\bar{x})=\nabla_{\nu}^{2}+\eta(\bar{x}) ;
$$

finally let $u$ and $v$ be differentiable functions defined on the union of $\Omega$ and $\partial \Omega$. The following identities hold.

Green's First Identity:

$\int_{\Omega} u * L[u] d \tau=\int_{\Omega}\left\{|\nabla u|^{2}-\left.\ln (\bar{x})\right|^{2}\right\} d \tau-\int_{\partial \Omega}(u * \nabla u) \cdot(\hat{n}) d \sigma$. 
Green's Second Identity:

$\int_{\Omega}\{u * L(v)-v(L[u]) *\} d \tau=\int_{\partial \Omega}(u * \nabla v-v \nabla u *) \cdot \hat{n} d \sigma$.

\subsection{THE FREDHOLM ALTERNATIVE THEOREM [6]}

The Boundary value problem

$$
\begin{aligned}
& L(\phi)=f(\bar{x}) \text { for all } \bar{x} \text { in } \Omega \\
& \phi(\bar{x})=0 \text { for all } \bar{x} \text { in } \partial \Omega
\end{aligned}
$$

has a unique solution only if the corresponding homogeneous problem has a nontrivial solution, $u \neq 0$, the inhomogeneous problem will have a solution only if $(u, f)=\int_{\Omega} u^{*} f d \tau=0$. In this case there is an infinity of solutions of the form $\phi=c u+\phi_{p}$, where $u$ satisfies the homogeneous problem and $\phi_{p}$ is any particular solution of the inhomogeneous problem.

\subsection{VARIOUS THEOREMS AND PROPOSITIONS}

THEOREM 1 [7] Suppose $\delta(\bar{x}-\bar{y})$ is the Dirac delta function for the specific n-dimensional coordinate system in use. Let $L$ be a self-adjoint linear differential operator of the form $L=\nabla_{n}^{2}+\eta(\bar{x})$.

(a) If the homogeneous problem

$$
\begin{aligned}
& \mathrm{L}[\mathrm{u}]=0 \text { in } \Omega \\
& \mathrm{u}=0 \text { on } \partial \Omega
\end{aligned}
$$

has only the trivial solution $u \equiv 0$, then there exists a unique solution (in the sense of generalized functions) to the problem

$$
\begin{aligned}
& L[g(\bar{x}, \bar{y})]=\delta(\bar{x}, \bar{y}) \text { for all } \bar{x} \text { and } \bar{y} \text { in } \Omega, \\
& g(\bar{x}, \bar{y})=0 \text { for all } \bar{x} \text { on } \partial \Omega \text {. }
\end{aligned}
$$

The function $g(\bar{x}, \bar{y})$ is called the Green's Function for the boundary value problem and is dependent not only on the operator $L$, but also on the geometry of $\Omega$ and its boundary. 
(b) If the homogeneous problem

$$
\begin{aligned}
& \mathrm{L}[\mathrm{u}]=0 \text { in } \Omega \\
& \mathrm{u}=0 \text { on } \partial \Omega,(\mathrm{u}, \mathrm{u})=1
\end{aligned}
$$

has a non-trivial solution $u(\bar{x}) \neq 0$, then there exists a (non-unique) solution to the problem

$$
L[G(\bar{x}, \bar{y})]=\delta(\bar{x}-\bar{y})-u *(\bar{y}) u(\bar{x}) \text { for all } \bar{x} \text { and } \bar{y} \text { in } \Omega \text {, and } G(\bar{x}, \bar{y})=0
$$

for all $\bar{x}$ on $\partial \Omega$.

The function $G(\bar{x}, \bar{y})$ is called the modified Green's function for the boundary value problem. It will be noted, that in keeping with the Fredholm Alternative Theorem, the generalized function $\rho(\bar{x}, \bar{y})=\delta(\bar{x}-\bar{y})-u *(\bar{y}) u(\bar{x})$ is orthogonal to $u(\bar{x})$. We have

$$
\begin{aligned}
(u, \rho) & =\int_{\Omega} u *(\bar{x}) \rho(\bar{x}, \bar{y}) d^{\nu} x=\int_{\Omega}[\delta(\bar{x}-\bar{y})-u *(\bar{y}) u(\bar{x})] u *(\bar{x}) d^{\nu} x \\
& =u *(\bar{y})-u(\bar{y})(u, u)=0,
\end{aligned}
$$

where we have assumed (as we may) that $u$ is normalized.

PROPOSITION 1 [8]: The Green's function in part (a) of Theorem 1 is symmetric in the sense that $g^{\star}\left(\bar{x}_{1}, \bar{x}_{2}\right)=g\left(\bar{x}_{2}, \bar{x}_{1}\right)$ for every $\bar{x}_{1}$ and $\bar{x}_{2}$ lying in $\Omega$.

PROPOSITION 2 [9]: Let $u$ be a solution of the completely homogeneous system: $L[u]=0$ for $a l l \bar{x}$ in $\Omega, u=0$ for all $\bar{x}$ in $\partial \Omega$. If we require that $(u, g)=0$, this is sufficient to insure that $g$ will be symetric.

THEOREM 2 [10]

(a) If the completely homogeneous problem has only the trivial solution, then the unique solution to

$$
L[\phi]=f(\bar{x}) \text { for all } \bar{x} \text { in } \Omega,
$$

and

$$
\phi(\bar{x})=0 \text { for all } \bar{x} \text { on } \partial \Omega
$$

is given by 


$$
\phi(\bar{x})=\int_{\Omega} g *(\bar{y}, \bar{x}) f(\bar{y}) d^{\nu} y=\int_{\Omega} g(\bar{x}, \bar{y}) f(\bar{y}) d^{\nu} y .
$$

Where $g(\bar{x}, \bar{y})$ is the Green's Function defined in part (a) of Theorem 1 .

(b) If the completely homogeneous problem has a non-trivial solution (say $u$ ), and if $(u, f)=0$, then all solutions of

$$
\begin{aligned}
& L \phi=f(\bar{x}) \text { for all } \bar{x} \text { in } \Omega, \\
& \phi(\bar{x})=0 \text { for all } \bar{x} \text { in } \partial \Omega
\end{aligned}
$$

are given by

$$
\phi(\bar{x})=\operatorname{Cu}(\bar{x})+\int_{\Omega} G *(\bar{y}, \bar{x}) f(\bar{y}) d^{\nu} y
$$

or

$$
\phi(\bar{x})=\operatorname{Cu}(\bar{x})+\int_{\Omega} G(\bar{x}, \bar{y}) f(\bar{y}) d^{\nu} y,
$$

where $C$ is an arbitrary constant.

\section{THE APPROXIMATION THEORY}

To begin we recall the basic equation from Section 1

$$
\mathrm{H}_{0} \phi_{f}=\mathrm{w}_{\mathrm{j}} \phi_{\mathrm{j}} ;
$$

the solution of which is known, and the equation of interest which can be written in two different ways,

$$
\left(H_{0}-E_{n}\right) \psi_{n}=-\varepsilon H_{1} \psi_{n} \text {, }
$$

or

$$
\left(H_{0}-W_{n}\right) \psi_{n}=\left(E_{n}-W_{n}-\varepsilon H_{1}\right) \psi_{n} \cdot
$$

\subsection{APPROXIMATION THEORY BASED ON EQUATION (3.2)}

We shall start with eq. (3.2) and apply Theorem 2 with $\mathrm{L}=\mathrm{H}_{0}-\mathrm{E}_{\mathrm{n}}$ and $f(\bar{x})=-\varepsilon H_{1} \psi_{n}$. We first see which part of the alternative will apply. The eq. $\left(\mathrm{H}_{0}-\mathrm{E}_{\mathrm{n}}\right) \psi_{\mathrm{n}}=0$ will have a non-trivial solution only if $\mathrm{E}_{\mathrm{n}}=\mathrm{W}_{\mathrm{s}}$ for some $\mathrm{s}$. To explore the consequences of this, we use the following equation:

$$
\left(E_{n}-W_{s}\right)\left(\phi_{s}, \Psi_{n}\right)=\varepsilon\left(\phi_{s}, H_{1} \Psi_{n}\right) .
$$


If $E_{n}=W_{s}$, then either $\varepsilon=0$ or $\left(\phi_{s}, H_{1} \psi_{n}\right)=0$. In the case of $\varepsilon=0$, we are back to the original unperturbed problem and we have $s=n, E_{n}=W_{n}$, and $\psi_{n}=\phi_{n}$. The case $E_{n}=W_{s}$ with $\varepsilon \neq 0$ and $s \neq n$ involves subtleties that have no bearing on the present development. [11] We, therefore, assume that $\varepsilon$ is such that $W_{n}<E_{n}<W_{n+1}$. With this assumption, $\left(H_{0}-E_{n}\right) \psi_{n}=0$ has only the trivial solution $\psi_{n}=0$, and by Theorem 2, part (a), $\psi_{n}$ is given by

$$
\psi_{n}(\bar{x})=-\varepsilon \int_{\Omega} g(\bar{x}, \bar{y}) H_{1}(\bar{y}) \psi_{n}(\bar{y}) d^{\nu} y
$$

The Green's Function, $g(\bar{x}, \bar{y})$, satisfies

$$
\left(H_{0}-E_{n}\right) g(\bar{x}, \bar{y})=\delta(\bar{x}-\bar{y}) \text { for all } \bar{x} \text { and } \bar{y} \text { in } \Omega
$$

and

$$
g(\bar{x}, \bar{y})=0 \text { for all } \bar{x} \text { on } \partial \Omega
$$

By standard techniques eq. $(2.3 .2)$ can be formed into an equation for $E_{n}$

$$
E_{n}=W_{n}+\varepsilon\left(\phi_{n}, H_{1} \psi_{n}\right) /\left(\phi_{n}, \psi_{n}\right) \text {. }
$$

We begin by supposing that system $(3.1 .2,3.1 .3)$ can be solved for $g(\bar{x}, \bar{y})$. Note that $E_{n}$ will occur as an unknown parameter, so it is more informative to write $g\left(\bar{x}, \bar{y} ; E_{n}\right)$. We then have the following system for $E_{n}$ and $\psi_{n}$ :

$$
\begin{aligned}
& \psi_{n}=-\varepsilon \int_{\Omega} g\left(\bar{x}, \bar{y} ; E_{n}\right) H_{1}(\bar{y}) \psi_{n}(\bar{y}) d^{\nu} y, \\
& E_{n}=w_{n}+\varepsilon\left(\phi_{n}, H_{1}, \psi_{n}\right) /\left(\phi_{n}, \psi_{n}\right),
\end{aligned}
$$

where $H_{1}(\bar{y}), \phi_{n}(\bar{x}), W_{n}$, and $g\left(\bar{x}, \bar{y} ; E_{n}\right)$ are presumed known. The strategy is to employ a method of successive approximation and solve the system $(3.1 .5,3.1 .6)$ self-consistently at any order of approximation. To show that this process is feasible, we prove two crucial results:

$$
\text { (i) } \lim _{\varepsilon \rightarrow 0} \psi=\phi_{n} \text {. }
$$

(This will assure that we can start the iteration and that we can begin with $\left.\psi_{\mathrm{n}}^{(0)}=\phi_{\mathrm{n}}\right)$ 
(11) The sequence defined by:

$$
\psi_{n}^{(k+1)}(\bar{x})=\varepsilon \int_{\Omega^{g}} g\left(\bar{x}, \bar{y} ; E_{n}\right) H_{1}(\bar{y}) \psi_{n}^{(k)}(\bar{y}) d^{\nu} y \quad(k \geq 0) .
$$

will converge for sultably restricted $\varepsilon$, thus insuring that we have a valid approximation procedure.

To facilitate these proofs, we want to expand $g\left(\bar{x}, \bar{y} ; E_{n}\right)$ in the complete set of eigenfunctions $\left\{\phi_{k}\right\}_{1}^{\infty}$. Let $g=\sum_{n=1}^{\infty} a_{k} \phi_{k}$ where the $a_{k}$ 's are to be determined. Substitute this into eq. (3.1.6) and obtain

$$
\sum_{k=1}^{\infty} a_{k}\left(W_{k}-E_{n}\right) \phi_{k}(\bar{x})=\delta(\bar{x}-\bar{y}) \text {, }
$$

where we have used $H_{0} \phi_{k}=w_{k} \phi_{k}$. Multiply by $\phi_{\ell}^{*}(\bar{x})$, integrate and note that $\left(\phi_{\ell}, \phi_{k}\right)=\delta_{\ell k}$ to obtain

$$
a_{k}=\phi_{k}^{*}(\bar{y}) /\left(w_{k}-E_{n}\right),
$$

giving the following series for $g$ :

$$
g\left(\bar{x}, \bar{y} ; E_{n}\right)=\sum_{k=1}^{\infty} \phi_{k}^{*}(\bar{y}) \phi_{k}(\bar{x}) /\left(w_{k}-E_{n}\right) \text {, with } E_{n} \neq w_{k} \cdot
$$

Using eq. (3.1.4), we obtain

$$
\begin{aligned}
g\left(\bar{x}, \bar{y} ; E_{n}\right)= & -\left(\phi_{n}, \psi_{n}\right) \phi_{n}^{*}(\bar{y}) \phi_{n}(\bar{x}) / \varepsilon\left(\phi_{n}, H_{1} \psi_{n}\right) \\
& +\sum_{k \neq n} \phi_{k}^{*}(\bar{y}) \phi_{k}(\bar{x}) /\left(W_{k}-E_{n}\right)
\end{aligned}
$$

Substitution of eq. (3.1.9) into eq. (3.1.5) produces

$$
\psi_{n}=\left(\phi_{n}, \psi_{n}\right) \phi_{n}(\bar{x})+\varepsilon \sum_{k \neq n}\left(\phi_{k}, H_{1}, \psi_{n}\right) /\left(E_{n}-W_{k}\right) .
$$

Let $\varepsilon \rightarrow 0$ and get $\psi_{n}^{(0)}=\left(\phi_{n}, \psi_{n}^{(0)}\right) \phi_{n}(\bar{x})$, 1.e., $\psi_{n}^{(0)}$ is a multiple of $\phi_{n}$. We shall employ the practice of requiring that $\left(\phi_{n}, \psi_{n}^{(k)}\right)=1$ at any stage of approximation, then normalizing at the end. This completes the proof of ( 1 ). We note that eqs. (3.1.4, 3.1.10) with $\left(\phi_{n}, \psi_{n}\right)=1$ are the standard expressions from which the Brillouin-Wigner Perturbation procedure is developed. [12, 13, 14] The connection between the integral representation of the solution in term of Green's 
Functions and the BW equations is thus apparent.

We now prove the second result. Let $\psi_{n}$ be the hypothetical function to which the $\psi_{n}^{(j)}$ are supposed to converge. If we can show that $\lim _{j \rightarrow \infty}\left\|\psi_{n}^{(j)}-\psi_{n}\right\|=0$, with the proper choice of $\varepsilon$, then we will have convergence of the iteration procedure. From eq. (3.1.7) we have the following expression:

$$
\psi_{n}^{(j)} \overline{(\bar{x})}=-\varepsilon \int_{\Omega} k_{1}\left(\bar{x}, \bar{y} ; E_{n}\right) \psi_{n}^{(j-1)}(\bar{y}) d^{\nu} y .
$$

The kernel, $k_{1}(\bar{x}, \bar{y})$, is defined by

$$
\mathrm{k}_{1}\left(\overline{\mathrm{x}}, \overline{\mathrm{y}} ; \mathrm{E}_{\mathrm{n}}\right) \equiv \mathrm{g}(\overline{\mathrm{x}}, \overline{\mathrm{y}}) \mathrm{H}_{1}(\overline{\mathrm{y}}) .
$$

We write eq. (3.1.11) in the more convenient form

$$
\psi_{n}^{(j)}=k_{1} \psi_{n}^{(j-1)}
$$

where $K_{1}[u] \equiv-\varepsilon \int g\left(\bar{x}, \bar{y} ; E_{n}\right) H_{1}(\bar{y}) u(\bar{y}) d^{\nu} y$.

It is clear, using eq. (3.1.13) and the linearity of $k_{1}$ that

$$
k_{1} \psi_{n}^{(j-1)}=k_{1}^{2} \psi_{n}^{(j-2)}=k_{1}^{3} \psi_{n}^{(j-3)}=\ldots=k_{1}^{j} \psi_{n}^{(0)}=k_{1}^{j} \phi_{n} .
$$

Now, since $\psi_{n}$ is supposed to be a solution for eq. (3.1.1), we have $k_{1}^{p} \psi_{n}=\psi_{n}$, for all integers $p \geq 0$. We can therefore write

$$
\left\|\psi_{n}^{(j)}-\psi_{n}\right\|=\left\|k_{1}^{j} \phi_{n}-k_{1}^{j} \psi_{n}\right\| \text {. }
$$

Using the linearity of $\mathrm{K}_{1}^{j}$ and the fact that $\phi_{n}$ and $\psi_{n}$ are fixed functions, we can write

$$
\left\|\psi_{n}^{(j)}-\psi_{n}\right\|=\left\|k_{1}^{j}\left(\phi_{n}-\psi_{n}\right)\right\|=\left\|k_{1}\right\|^{j}\left\|\phi_{n}-\psi_{n}\right\|
$$

It is clear from eq. (3.1.16) that $\lim _{j \rightarrow \infty}|| \psi_{n}^{(j)}-\psi_{n} \|=0$ if and only if $\left\|k_{1}\right\|<1$.

Using the inequality ||$k||^{2} \leq \iint|k(\bar{x}, \bar{y})|^{2} d^{v} \bar{x} d^{\nu} y$ for Hilbert Schmidt

operators [15] and the definition of $\mathrm{K}_{1}$, we impose the following requirements:

$$
\left\|\mathrm{k}_{1}\right\|^{2} \leq \varepsilon^{2} \iint_{\Omega \Omega}\left|\mathrm{k}_{1}\left(\overline{\mathrm{x}}, \overline{\mathrm{y}} ; \mathrm{E}_{\mathrm{n}}\right)\right|^{2} \mathrm{~d}^{\nu} \mathrm{x} \mathrm{d}^{\nu} \mathrm{y}<1
$$


We therefore have the following inequality for $\varepsilon$ :

$$
|\varepsilon|<\left\{\int_{\Omega} \int_{\Omega}\left|k_{1}\left(\bar{x}, \bar{y} ; E_{n}\right)\right|^{2} d^{\nu} x d^{v} \bar{y}\right\}^{-\frac{1}{2}} \text {. }
$$

Thus we have shown that for some range of $\varepsilon$, we have a reasonable approximation procedure. Since the inequality $(3.1 .18)$ implicitly contains the unknown energy, $E_{n}$, on the right side it is not useful as a practical estimate of the convergence radius. However, its use as a demonstration of convergence in principle is not compromised by the occurrence of the unknown $E_{n}$ provided, as assumed, $E_{n} \neq W_{k}$ for any $k$. This can be seen by a reference to equation (3.1.8) and the definition of $k_{1}\left(\bar{x}, \bar{y} ; E_{n}\right)$. Whatever the energy $E_{n}$ is, as long as $E_{n} \neq w_{k}$, the integral on the right of inequality (3.1.18) will be a finite number $>0$ and thus an $\varepsilon$ can be found which will satisfy (3.1.18).

The approximation scheme is summarized below:

$$
\psi_{n}^{(k+1)}(\bar{x})=-\varepsilon \int_{\Omega} g\left(x, y ; E_{n}^{(k)}\right) H_{1}(\bar{y}) \psi_{n}^{(k)}(\bar{y}) d^{\nu} y
$$

and

$$
\mathrm{E}_{\mathrm{n}}^{(\mathrm{k}+1)}=\mathrm{w}_{\mathrm{n}}+\left(\phi_{\mathrm{n}}, \mathrm{H}_{1}, \psi_{\mathrm{n}}^{(\mathrm{k})}\right), \text { with } \mathrm{k}=0,1,2, \ldots
$$

The occurrence of $E_{n}$ as an unknown parameter on the RHS of both equations means that at any given level of approximation the system will have to be solved selfconsistently by numerical procedure. Although this makes the calculation more difficult, it has the potential of returning some quite good values for $E_{n}$ with a fairly low order approximation to $\psi_{n}$.

\subsection{APPROXIMATION PROCEDURE BASED ON EQUATION (3.3)}

We observe that the homogeneous equation, $\left(H_{0}-W_{n}\right) \psi_{n}=0$, has a non-trivial solution; namely, $\phi_{n}$. Thus Theorem 2, part (b) applies, and we can write the solution to eq. (3.1) as

$$
\psi_{n}=C \phi_{n}+\int_{\Omega} G(\bar{x}, \bar{y})\left[E_{n}-W_{n}-\varepsilon H_{\overline{1}}\right] \psi_{n}(\bar{y}) d^{\nu} y,
$$


Provided that $\left(\phi_{n},\left[E_{n}-W_{n}-\varepsilon H_{1}\right] \psi_{n}\right)=0$, which is the same as

$$
\left(E_{n}-W_{n}\right)\left(\phi_{n}, \varepsilon H_{1} \psi_{n}\right) \text {. }
$$

Equation (3.2.2) is satisfied, as has already been noted.

Recall also, that $G(\bar{x}, \bar{y})$ must satisfy

$$
\left(\mathrm{H}_{0}-\mathrm{W}_{\mathrm{n}}\right) G(\overline{\mathrm{x}}-\overline{\mathrm{y}})=\delta(\overline{\mathrm{x}}-\overline{\mathrm{y}})-\phi_{\mathrm{n}}^{*}(\overline{\mathrm{y}}) \phi_{\mathrm{n}}(\overline{\mathrm{x}}) \text {. }
$$

Following the convention adopted in Section 3.1., we shall require that $\left(\phi_{n}, \psi_{n}^{(k)}\right)=1$ at each order of the approximation and normalize at the end. With this convention, we can replace $E_{n}-W_{n}$ in Eq. (3.2.1) and obtain

$$
\psi_{n}=C \phi_{n}+\varepsilon\left(\phi_{n}, H_{1} \psi_{n}\right) \int_{\Omega} G(\bar{x}, \bar{y}) \psi_{n}(\bar{y}) d^{\nu} y-\varepsilon \int_{\Omega} G(\bar{x}, \bar{y}) H_{1}(y) \psi_{n}(\bar{y}) d^{\nu} y .
$$

As a preliminary result, we now show that $\left(\phi_{n}, \psi_{n}\right)=1$ if and only if $c=1$.

PROOF: Multiply eq. $(3.2 .4)$ by $\phi_{n}^{*}(x)$ and integrate to get:

$$
\begin{aligned}
\left(\phi_{n}, \psi_{n}\right)= & c+\varepsilon\left(\phi_{n}, H_{1} \psi_{n}\right) \int_{\Omega} \int_{\Omega} \phi_{n}^{*}(\bar{x}) G(\bar{x}, \bar{y}) \psi_{n}(\bar{y}) d^{v} \bar{y}^{\nu} x \\
& -\varepsilon \int_{\Omega} \int_{\Omega} \phi_{n}^{*}(\bar{x}) G(x, y) H_{1}(\bar{y}) \psi_{n}(\bar{y}) d^{v} \bar{y}^{v} \bar{x}^{2} .
\end{aligned}
$$

By Proposition 2, we have $\left(\phi_{n}, G\right)=0$ for every $\bar{y}$ in $\Omega$, giving $C=\left(\phi_{n}, \psi_{n}\right)$. We therefore write

$$
\begin{aligned}
\psi_{n}(\bar{x})= & \phi_{n}(\bar{x})+\varepsilon\left(\phi_{n}, H_{1} \psi_{n}\right) \int_{\Omega} G(x, y) \psi_{n}(y) d^{\nu} y \\
& -\varepsilon \int_{\Omega} G(\bar{x}, \bar{y}) H_{1}(\bar{y}) \psi_{n}(\bar{y}) d^{\nu} \bar{y} .
\end{aligned}
$$

Equation (3.2.5) will be the basis for a useful approximation procedure. We begin by proving the following proposition:

PROPOSITION 3: Suppose that $G(\bar{x}, \bar{y}), H_{1}(\bar{y})$ and $\psi_{n}(x ; \varepsilon)$ satisfy the following hypotheses:

(i) $G(\bar{x}, \bar{y})$ is bounded and continuous on $\Omega \times \Omega$

(ii) $\psi_{n}(x ; \varepsilon)$ is well-defined for all $\varepsilon$ contained in $N_{\delta} \equiv\{\varepsilon|0 \leq| \varepsilon \mid<\delta\}$ and is bounded and continuous with respect to $\bar{x}$ on $\Omega$ 
(iii) $\mathrm{H}_{1}(\bar{y}), \mathrm{H}_{1}(\bar{y}) G(\bar{x}, \bar{y})$, and $\mathrm{H}_{1}(\bar{y}) G(\bar{x}, \bar{y}) \psi_{n}(x, \varepsilon)$ are Reiman Integrable with respect to $\bar{y}$ for all $\bar{x}$ in $\Omega$ and for all $\varepsilon$ in $N_{\delta}$.

Then, $\psi_{n}(x ; \varepsilon)$ is continuous with respect to $\varepsilon$ at $\varepsilon=0$, and $\lim _{\varepsilon \rightarrow 0} \psi_{n}(\bar{x} ; \varepsilon)=\phi_{n}(\bar{x})$.

PROOF: Using eq. $(3.2 .5)$, we write

$$
\psi_{n}(x ; \varepsilon)=\phi_{n}(\bar{x})+\varepsilon\left(\phi_{n}, H_{1} \psi_{n}\right) \int_{\Omega} G(\bar{x}, \bar{y}) \psi_{n}(\bar{y} ; \varepsilon) d^{\nu} \bar{y}-\varepsilon \int_{\Omega} G(\bar{x}, \bar{y}) H_{1}(\bar{y}) \psi_{n}(x ; \varepsilon) d^{\nu} \bar{y} .
$$

Using this and the postulated properties of $\mathrm{H}_{1}, G$, and $\psi_{n}$, it is clear that $\psi_{n}(x ; 0)=\phi_{n}(\bar{x})$. Next we show that $\lim _{\varepsilon \rightarrow 0} \psi_{n}(x ; \varepsilon)=\phi_{n}(\bar{x})$, proving continuity. We have

$$
\left|\psi_{n}-\phi_{n}\right| \leq|\varepsilon|\left\{\left|\int_{\Omega} G \psi_{n} d^{v}-\int_{\Omega} G H_{1} \psi_{n} d^{v} y\right|\right\} .
$$

By hypothesis, there must exist $M<\infty$ such that $\left|\int_{\Omega} G \psi_{n} d^{\nu} \bar{y}-\int_{\Omega} G H_{1} \psi_{n} d^{\nu} y\right| \leq M$, and we get $\left|\psi_{n}-\phi_{n}\right| \leq|\varepsilon M|$, or $\lim _{\varepsilon \rightarrow 0} \psi_{n}(x ; \varepsilon)=\phi_{n}(\bar{x})$, concluding the proof.

As the next step, we have

PROPOSITION 4: Under the same hypotheses as Proposition $3,\left.\left[\partial \psi_{\mathbf{n}} / \partial \varepsilon\right]\right|_{\varepsilon=0}$ exists for all $\bar{x}$ in $\Omega$.

PROOF: Consider the difference $\psi_{n}(x ; h)-\psi_{n}(x ; 0)$ and use Proposition 3 to obtain

$$
\begin{aligned}
\psi_{n}(\bar{x} ; h)-\psi_{n}(\bar{x} ; 0)= & h\left\{\left[\left(\phi_{n}, H_{1} \psi_{n}(\bar{s} ; h)\right)\right] \int_{\Omega} G(\bar{x}, \bar{y}) \psi_{n}(\bar{y} ; h) d^{\nu} y\right. \\
& \left.-\int_{\Omega} G(\bar{x}, \bar{y}) H_{1} \psi_{n}(\bar{y} ; h) d^{\nu} y\right\} .
\end{aligned}
$$

The equation being valid and well-defined for $0 \leq h \leq \delta$. We now divide both sides of eq. (3.2.6) by $h$, and consider the limit of the RHS as $h \rightarrow 0$. We obtain

$$
\lim _{h \rightarrow 0}\left[\left(\phi_{n}, H_{1} \psi_{n}\right) \int_{\Omega} G \psi_{n} d^{\nu} y-\int_{\Omega} G H_{1} \psi_{n} d^{\nu} y\right]=-\left(G, H_{1} \phi_{n}\right)(\bar{x})
$$

since using Proposition 2 and 3

$$
\int_{\Omega} G(\bar{x}, \bar{y}) \phi_{n}(\bar{y}) d^{\nu} y=0 .
$$

Since the limit as $h \rightarrow 0$ of the RHS/h of eq. (3.2.6) exists, so also must limit of 
LHS/h as $h \rightarrow 0$, implying that $\left.\left[\partial \Psi_{n}(x ; \varepsilon) / \partial \varepsilon\right]\right|_{\varepsilon=0}$ exists and is in fact given by

$$
\partial \psi_{n}(\bar{x} ; \varepsilon) /\left.\partial \varepsilon\right|_{\varepsilon}=0-\int_{\Omega} G(\bar{x}, \bar{y}) H_{1}(\bar{y}) \phi_{n}(\bar{y}) d^{\nu} y .
$$

We now prove the following proposition:

PROPOSITION 5: The derivative, $\left.\left[\partial^{k} \psi_{n}(\bar{x} ; \varepsilon) / \partial \varepsilon^{k}\right]\right|_{\varepsilon=0}$ exists for all $k \geq 0$, under the same assumptions as Propositions 3 and 4 .

PROOF: We shall prove this by induction: Proposition 3 and 4 are the cases $k=0$ and $k=1$. We write eq. $(3.2 .6)$ as follows:

$$
\psi_{n}(\bar{x})=\phi_{n}(\bar{x})+\varepsilon F(\bar{x} ; \varepsilon),
$$

where $F$ is defined as

$$
F(\bar{x}, \varepsilon) \equiv\left(\phi_{n}, H_{1} \psi_{n}\right) \int_{\Omega} G(\bar{x}, \bar{y}) \psi_{n}(\bar{y}) d^{\nu} y-\int_{\Omega} G(\bar{x}, \bar{y}) H_{1}(\bar{y}) \psi_{n}(\bar{y}) d^{\nu} y
$$

We assume that $\left.\left[\partial^{k} \psi_{n} / \partial \varepsilon^{k}\right]\right|_{\varepsilon=0}$ exists for $k=0,1,2, . ., \ell$ and show the existence for $\ell+1$. The existence of $\left.\left[\partial^{\ell} \psi_{n} / \partial \varepsilon^{\ell}\right]\right|_{\varepsilon=0}$ implies the existence of $\left.\left[\partial^{\ell} F / \partial \varepsilon^{\ell}\right]\right|_{\varepsilon=0}$. In fact we have, using Liebnitz' Rule for the derivative of a product [16]

$$
\begin{aligned}
\partial^{\ell} F / \partial \varepsilon^{\ell}= & \left\{\sum_{j=0}^{\ell} C_{\ell, j}\left(\phi_{n}, H_{1} \partial^{\ell-j} \psi_{n} / \partial \varepsilon^{\ell-j}\right) \int_{\Omega} G(\bar{x}, \bar{y})\left(\partial^{j} \psi_{n} / \partial \varepsilon^{j}\right) d^{\nu} y\right. \\
& -\left.\int_{\Omega} G(\bar{x}, \bar{y}) H_{1}(\bar{y})\left(\partial^{\ell} \psi_{n} / \partial \varepsilon^{\ell}\right) d^{\nu} y\right|_{\varepsilon=0} .
\end{aligned}
$$

Clearly, the RHS of eq. (3.2.8) depends only on the existence of $\left.\left[\partial^{k} \psi_{n} / \partial \varepsilon^{k}\right]\right|_{\varepsilon=0}$, for $k=0,2, \ldots, \ell$. Next, observe that

$$
\begin{aligned}
\partial^{\ell} \psi_{\mathrm{n}} /\left.\partial \varepsilon^{\ell}\right|_{\varepsilon=0} & =\left.\left(\partial^{\ell} / \partial \varepsilon^{\ell}\right)[\varepsilon \mathrm{F}(\overline{\mathrm{x}} ; \varepsilon)]\right|_{\varepsilon=0}=\ell \partial^{\ell-1} \mathrm{~F} /\left.\partial \varepsilon^{\ell}\right|_{\varepsilon=0}+\varepsilon \partial^{\ell} F /\left.\partial \varepsilon^{\ell}\right|_{\varepsilon=0} \\
& =\ell \partial^{\ell-1} \mathrm{~F} /\left.\partial \varepsilon^{\ell-1}\right|_{\varepsilon=0} \cdot
\end{aligned}
$$

Finally, we show that eq. (3.2.9) holds for $\ell+1$. 
Consider

$$
\begin{aligned}
& \left.\partial^{l} \psi_{\mathrm{n}} \overline{(\bar{x}} ; \mathrm{h}\right) / \partial \varepsilon^{l}-\partial^{l} \psi_{\mathrm{n}}(\overline{\mathrm{x}} ; 0) / \partial \varepsilon^{\ell}= \\
& \ell \partial^{\ell-1} \mathrm{~F}(\overline{\mathrm{x}} ; \mathrm{h}) / \partial \varepsilon^{\ell}+\mathrm{h} \partial^{\ell} \mathrm{F}(\overline{\mathrm{x}} ; 0) / \partial \varepsilon^{\ell} \ell \partial^{\ell-1} \mathrm{~F}(\overline{\mathrm{x}} ; \mathrm{h}) / \partial \varepsilon^{\ell} .
\end{aligned}
$$

Divide eq. $(3.2 .10)$ by $h$ and consider the limit of the RHS as $h \rightarrow 0$ (presuming $\partial^{\ell} \mathrm{F}(\overline{\mathrm{x}} ; 0) / \partial^{\ell}$ to exist) we have

$$
\begin{aligned}
\partial^{l+1} \psi_{n}(\bar{x} ; 0) / \partial \varepsilon^{\ell+1} & \equiv \lim _{h \rightarrow 0}(1 / h)\left[\partial^{l} \psi_{n}(\bar{x} ; h)-\partial^{l} \Psi_{n}(\bar{x} ; 0) / \partial \varepsilon^{l}\right] \\
& =(l+1) \partial^{l} F(\bar{x} ; 0) / \partial \varepsilon^{l} .
\end{aligned}
$$

This concludes the proof of Proposition 5. We have shown that the function $\psi_{\mathrm{n}}(\overline{\mathrm{x}} ; \varepsilon)$ is $\mathrm{C}^{\infty}$ with respect to $\varepsilon$ at $\varepsilon=0$. Although this is a remarkable result, it does not prove that $\psi_{n}(\bar{x} ; \varepsilon)$ is analytic in $\varepsilon$.

From a practical point of view, however, Proposition 5 amply justifies trying the following power series expansion as a representation for $\psi_{n}(\bar{x} ; \varepsilon)$ :

$$
\psi_{n}(\bar{x} ; \varepsilon)=\phi_{n}(\bar{x})+\left.\varepsilon\left[\partial \psi_{n} / \partial \varepsilon\right]\right|_{\varepsilon=0}+\left.\left(\varepsilon^{2} / 2 !\right)\left[\partial^{2} \psi_{n} / \partial \varepsilon^{2}\right]\right|_{\varepsilon=0}+\ldots
$$

We next estimate the range of values of $\varepsilon$ for which the series (3.2.11) can be expected to converge. A dominating series is the following:

$$
s(\varepsilon)=\left\|\phi_{n}\right\|+|\varepsilon|\left\{|| \partial \psi_{n}(\bar{x} ; 0) / \partial \varepsilon||\right\}+\ldots
$$

We can write the series $(3.2 .12)$ in the simpler form

$$
s(\varepsilon)=\alpha_{0}+|\varepsilon| \alpha_{1}+\left|\varepsilon^{2} / 2 !\right| \alpha_{2}+\ldots
$$

where the definition of the $\alpha_{k}$ 's is obvious. By the ratio test, if $\lim _{\mathrm{p} \rightarrow \infty}\left|\varepsilon^{\mathrm{p}+1} \alpha_{\mathrm{p}+1} \mathrm{p} ! / \varepsilon^{\mathrm{p}} \alpha_{\mathrm{p}}(\mathrm{p}+1) !\right|<1$, then the series $s(\varepsilon)$ will be convergent, implying that the series (3.2.11) is absolutely and uniformly convergent for $\bar{x}$ in $\Omega$. Thus, let $\mathrm{L} \equiv \lim _{\mathrm{p} \rightarrow \infty}\left|\alpha_{\mathrm{p}+1} /(\mathrm{p}+1) \alpha_{\mathrm{p}}\right|$ and convergence will be assured if $|\varepsilon|<1 / \mathrm{L}$. In practice, the limit $L$ is hard to compute, so we will estimate it using the first 
two terms of the series, i.e., $p=0$. If we define

$$
K_{2}[u]=\int_{\Omega} G(\bar{x}, \bar{y}) H_{1}(\bar{y}) u(\bar{y}) d^{\nu} y,
$$

then we have

$$
\mathrm{L}=\alpha_{1} / \alpha_{0}=\left\|\mathrm{K}_{2}\left[\phi_{\mathrm{n}}\right]\right\| /\left\|\phi_{\mathrm{n}}\right\|=\left\|\mathrm{K}_{2}\right\| .
$$

Our estimate, therefore, becomes

$$
|\varepsilon|<\left\{\int_{\Omega} \int_{\Omega}\left|G(\bar{x}, \bar{y}) H_{1}(\bar{y})\right|^{2} d^{\nu} x d^{\nu} y\right\}^{-\frac{1}{2}} .
$$

Inequality (3.2.14) should be compared with the estimate (3.1.18) which pertained to the BW theory.

Our next task will be to show the connection of this procedure to the Rayleigh-Schrödinger theory. To do this, we expand $G(\bar{x}, \bar{y})$ in the orthonormal set $\left\{\phi_{k}\right\}^{\infty}{ }_{1}$.

Recall eq. (3.2.3)

$$
\left(\mathrm{H}_{0}-\mathrm{w}_{\mathrm{n}}\right) G(\overline{\mathrm{x}}, \overline{\mathrm{y}})=\delta(\overline{\mathrm{x}}-\overline{\mathrm{y}})-\phi_{\mathrm{n}}^{*}(\overline{\mathrm{y}}) \phi_{\mathrm{n}}(\overline{\mathrm{x}}) .
$$

Substitution of $G=\sum_{k=1} a_{k}{ }^{(n)} \phi_{k}(\bar{x})$, and multiplication by $\phi_{p}^{*}(\bar{x})$ followed by integration gives

$$
\sum_{k=1}^{\infty}\left(w_{k}-W_{n}\right) a_{k}^{(n)} \delta_{p k}=\phi_{p}^{*}(\bar{y})-\phi_{n}^{*}(\bar{y}) \delta_{p n},
$$

for calculating $a_{k}^{(n)}$.

We, therefore, write

$$
G(\bar{x}, \bar{y})=\sum_{s \neq n} \phi_{s}^{*}(\bar{y}) \phi_{s}(\bar{x}) /\left(w_{s}-w_{n}\right) .
$$

Substitution of eq. (3.2.16) into eq. (3.2.10) gives

$$
\begin{aligned}
\psi_{n}(\bar{x})= & \phi_{n}(\bar{x})+\varepsilon\left(\phi_{n}, H_{1} \psi_{n}\right) \sum_{s \neq n}\left(\phi_{s}, \psi_{n}\right) \phi_{s}(\bar{x}) /\left(W_{s}-W_{n}\right) \\
& -\varepsilon \sum_{s \neq n}\left(\phi_{s}, H_{1} \psi_{n}\right) \phi_{s}(\bar{x}) /\left(W_{s}-W_{n}\right) .
\end{aligned}
$$

Let $\psi_{n} \rightarrow \phi_{n}$ on the RHS of eq. (3.2.17) producing 


$$
\psi_{n}(1)=\phi_{n}+\varepsilon \sum_{s \neq n}\left(\phi_{s}, H_{1} \psi_{n}\right) \phi_{s}(\bar{x}) /\left(W_{n}-W_{s}\right)
$$

which is the defining equation for Rayleigh-Schrodinger Perturbation Theory $[13,14]$. Resubstitution of $\psi_{\mathrm{n}}{ }^{(1)}, \psi_{\mathrm{n}}{ }^{(1)}, \psi_{\mathrm{n}}{ }^{(3)}, \ldots \ldots \psi_{\mathrm{n}}{ }^{(\mathrm{k})}$ will produce the RS theory.

\section{SUMMARY}

\subsection{WAVE FUNCTION (EXACT - GREEN'S FUNCTION KERNELS)}

$$
\begin{aligned}
& \text { (BW) } \psi_{n}(\bar{x})=-\varepsilon \int_{\Omega} g_{B W}(\bar{x}, \bar{y}) H_{1}(\bar{y}) \psi_{n}(\bar{y}) d^{\nu} y \\
& \text { (RS) } \psi_{n}(\bar{x})=\phi_{n}+\int_{\Omega}\left[E_{n}-W_{n}-\varepsilon H_{1}(\bar{y})\right] g_{R S}(\bar{x}, \bar{y}) \psi_{n}(\bar{y}) d^{\nu} y
\end{aligned}
$$

The Green's Functions satisfy the following equations:

(BW) $\quad\left(H_{0}-E_{n}\right) g_{B W}(\bar{x}, \bar{y})=\delta(\bar{x}-\bar{y})$,

(RS) $\quad\left(\mathrm{H}_{0}-\mathrm{W}_{\mathrm{n}}\right) \mathrm{g}_{\mathrm{RS}}(\overline{\mathrm{x}}, \overline{\mathrm{y}})=\delta(\overline{\mathrm{x}}-\overline{\mathrm{y}})-\phi_{\mathrm{n}}^{*}(\overline{\mathrm{y}}) \phi_{\mathrm{n}}(\overline{\mathrm{x}})$,

\subsection{WAVE FUNCTIONS (EXACT - EIGENFUNCTION EXPANSION)}

$$
\text { (BW) } \begin{aligned}
\psi_{n}(\bar{x})= & \phi_{n}(\bar{x})-\varepsilon \sum_{k \neq n}\left(\phi_{n}, H_{1} \psi_{n}\right) /\left(W_{k}-E_{n}\right) \\
\text { (RS) } \psi_{n}(\bar{x})= & \phi_{n}(\bar{x})-\varepsilon\left(\phi_{n}, H_{1} \psi_{n}\right) \sum_{k \neq n}\left(\phi_{k}, \psi_{n}\right) \phi_{k}(\bar{x}) /\left(W_{k}-W_{n}\right) \\
& -\varepsilon \sum_{k \neq n}\left(\phi_{n}, H_{1} \psi_{n}\right) \phi_{k}(\bar{x}) /\left(W_{k}-W_{n}\right)
\end{aligned}
$$

\subsection{WAVE FUNCTIONS (FIRST ORDER - GREEN'S FUNCTION KERNELS)}

$$
\begin{aligned}
& \text { (BW) } \psi_{n}{ }^{(1)}=\phi_{n}-\varepsilon \int_{\Omega} g_{B W}\left(x, y ; E_{n}{ }^{(1)}\right) H_{1}(\bar{y}) \phi_{n}(\bar{y}) d^{\nu} y \\
& \text { (RS) } \psi_{n}{ }^{(1)}=\phi_{n}-\varepsilon \int_{\Omega} g_{R S}(\bar{x}, \bar{y}) H_{1}(\bar{y}) \phi_{n}(\bar{y}) d^{\nu} \bar{y}
\end{aligned}
$$

\subsection{WAVE FUNCTIONS (FIRST ORDER - EIGENFUNCTION EXPANSIONS)}

$$
\begin{aligned}
& \text { (BW) } \psi_{n}(1)=\phi_{n}-\varepsilon \sum_{k \neq n}\left(\phi_{k}, H_{1} \phi_{n}\right) \phi_{k}(\bar{x}) /\left(W_{k}-E_{n}\right) \\
& \text { (RS) } \psi_{n}(1)=\phi_{n}-\varepsilon \sum_{k \neq n}\left(\phi_{k}, H_{1} \phi_{n}\right) \phi_{k}(\bar{x}) /\left(W_{k}-W_{n}\right)
\end{aligned}
$$




\subsection{ENERGY FORMULAS (EXACT)}

$(B W, R S) \quad E_{n}=W_{n}+\varepsilon\left(\phi_{n}, H_{1} \psi_{n}\right)$

\section{APPENDIX--THE PERTURBED INFINITE SQUARE WELL IN CLOSED FORM PERTURBATION} THEORY. [8]

Consider the infinite square well of width L with a perturbation " $v_{0}$ " added to the right half of the well. The unperturbed solutions are

$$
\phi_{n}(x)=\sqrt{2 / L} \sin (n \pi x / L)
$$

and

$$
W_{n}=n^{2} h^{2} / 8 m L^{2} \text {. }
$$

The standard (RS) perturbation theory approach to this problem produces the following results for the ground state

$$
\mathrm{E}_{1}^{(2)}=\mathrm{w}_{1}+\mathrm{v}_{0} / 2-\left(r \mathrm{v}_{0}^{2} / \pi^{2} \mathrm{w}_{1}\right) \sum_{\mathrm{k}=1}^{\infty} \frac{4 \mathrm{k}^{2}}{\mathrm{k}^{2}-1}
$$

and

$$
\psi_{1}^{(1)}(\mathrm{x})=\phi_{1}(\mathrm{x})-\left(4 \mathrm{~V}_{0} / \mathrm{W}_{1} \pi\right) \sum_{\mathrm{k}=1}^{\infty}(-1)^{\mathrm{k}} \mathrm{k} \phi_{2 \mathrm{k}} /\left(4 \mathrm{k}^{2}-1\right)^{2} .
$$

The closed form modified Green's Function obtained by standard techniques is

$$
\begin{aligned}
G(x, y)= & \left(\mu / \lambda_{n} L\right)\left[(L-y) \cos \left(\lambda_{n} y\right) \sin \left(\lambda_{n} x\right)\right. \\
& \left.-x \sin \left(\lambda_{n} y\right) \cos \left(\lambda_{n} x\right)\right] \text { for } x<y
\end{aligned}
$$

and

$$
\begin{aligned}
G(x, y)= & \left(\mu / \lambda_{n} L\right)\left[(L-x) \cos \left(\lambda_{n} x\right) \sin \left(\lambda_{n} y\right)\right. \\
& \left.-y \sin \left(\lambda_{n} x\right) \cos \left(\lambda_{n} y\right)\right] \text { for } x>y
\end{aligned}
$$

where $\lambda_{n} \equiv n \pi / L$ and $\mu=2 m / h^{2}$. For the ground state, $n=1$, the use of this Green's Function in the appropriate equations yields

$$
\mathrm{E}_{1}^{(2)}=\mathrm{w}_{1}+\mathrm{v}_{0} / 2-\mathrm{v}_{0} / 16 \mathrm{w}_{1}
$$

and 


$$
\psi_{1}^{(1)}(\mathrm{x})=\phi_{1}(\mathrm{x})+\left(\mu \mathrm{v}_{0} / 4 \pi_{1}\right) \sqrt{2 / \mathrm{L}}\left\{\begin{array}{l}
x \cos \left(\lambda_{1} \mathrm{x}\right) \quad(\mathrm{x}<\mathrm{L} / 2) \\
(\mathrm{L}-\mathrm{x}) \cos \left(\lambda_{1} \mathrm{x}\right)(\mathrm{x}>\mathrm{L} / 2) .
\end{array}\right.
$$

These equations are equivalent [8] to equations (5.1) and (5.2) and in many cases would be more useful.

\section{REFERENCES}

1. Jackson, J. D., Classical Electrodynamics, John Wiley, New York, 1962.

2. Arfken, G., Mathematical Methods for Physicists, Academic Press, New York, $1970,748-766$.

3. Merzbacher, E., Quantum Mechanics, John Wiley, New York, 1970, Chapter 19.

4. Kato, T., Perturbation Theory for Linear Operators, Springer-Verlag, New York, 1976.

5. Rellich, F., Perturbation Theory of Eigenvalue Problems, Gordon and Breach, New York, 1969.

6. Korn, G. A. and T. M. Korn, Mathematical Handbook for Scientists and Engineers, McGraw-Hill, New York, 1968, 506.

7. Ibid., 515-516.

8. Rose, 0. J., A Study of Various Perturbation Methods for Solving the Time Independent Schrödinger Equation, M S Thesis, East Carolina University, 1975, 18-20.

9. Ibid.

10. Ibid.

11. Ibid, 104 .

12. Merzbacher, op cit.

13. Davydov, A. A., Quantum Mechanics, Neo Press, Ann Arbor, 1966, 171-173.

14. Silvert, W., Comparison of Rayleigh-Schrodinger and Brillouin-Wigner Perturbation Theories, American Journal of Physics, 40(1972).

15. Statgold, I., Boundary Value Problems of Mathematical Physics, Vol. I, MacMillan, New York, 1967.

16. Neilsen, K. L., Differential Equations, Barnes \& Noble, New York, $1962,11$. 


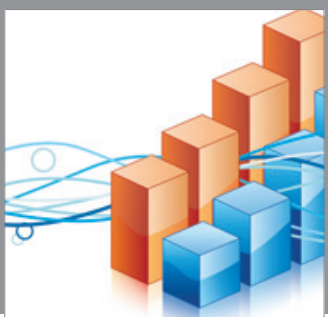

Advances in

Operations Research

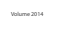

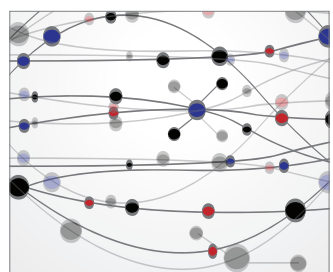

\section{The Scientific} World Journal
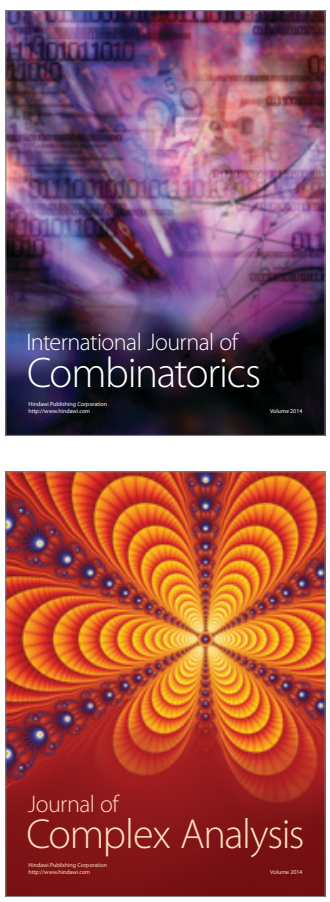

International Journal of

Mathematics and

Mathematical

Sciences
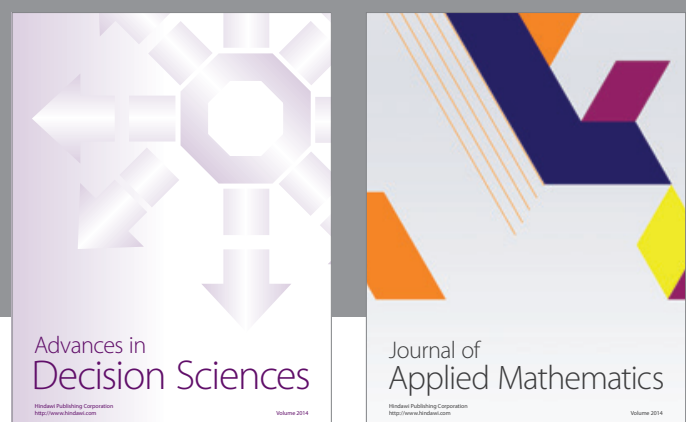

Journal of

Applied Mathematics
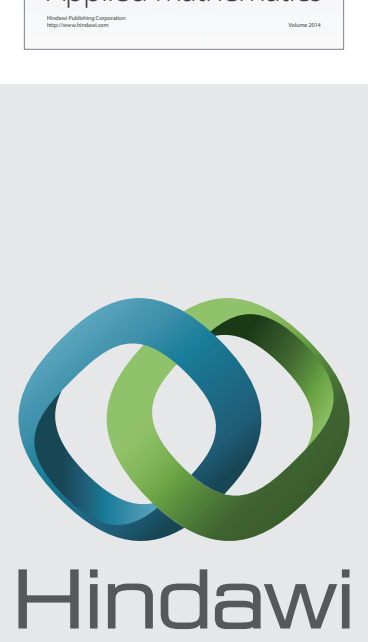

Submit your manuscripts at http://www.hindawi.com
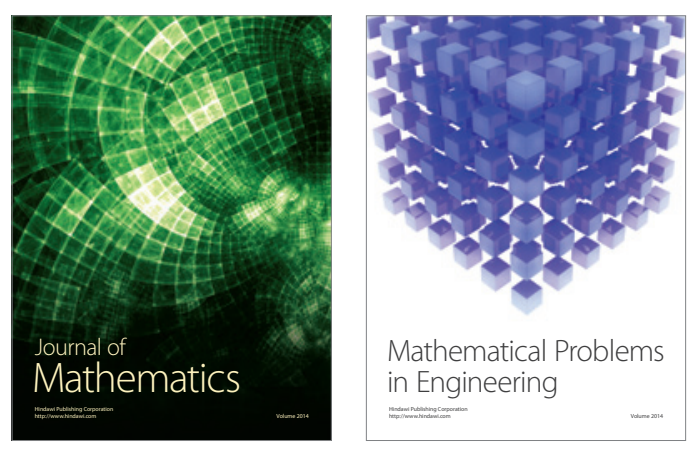

Mathematical Problems in Engineering
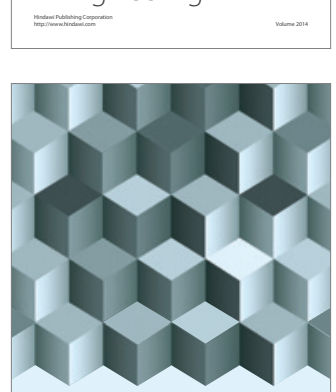

Journal of

Function Spaces
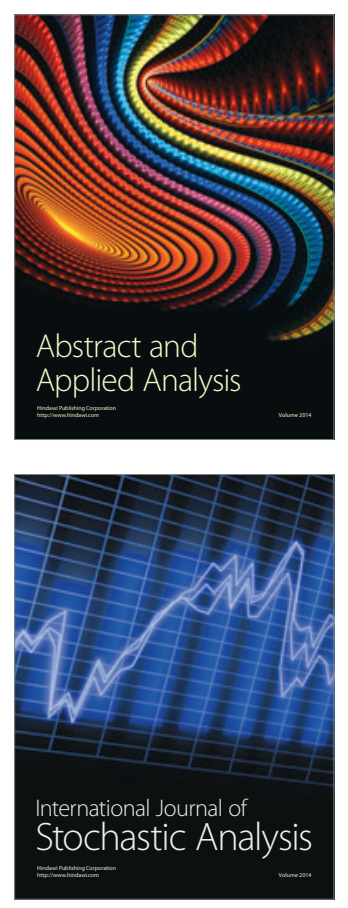

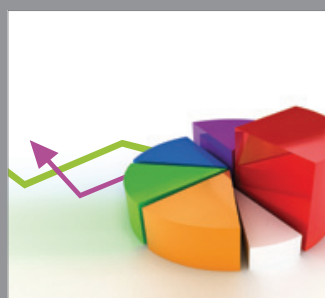

ournal of

Probability and Statistics

Promensencen
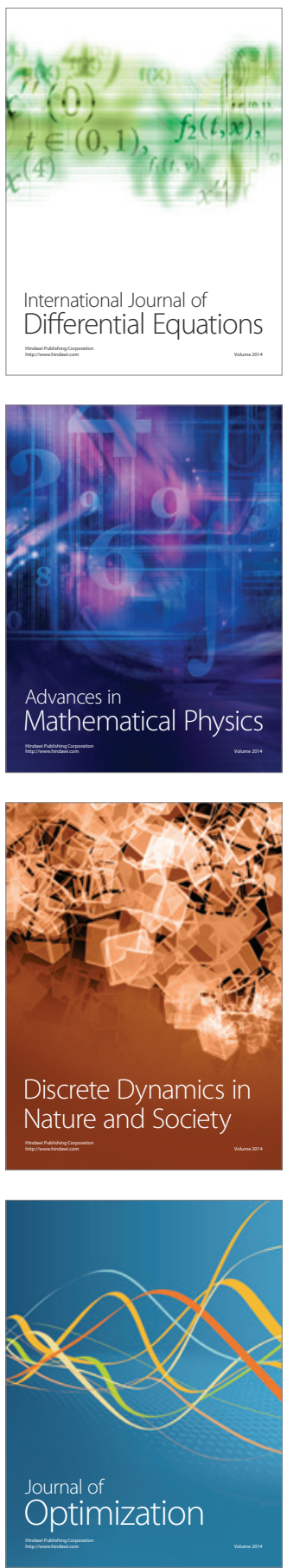